$\xi=2$

\title{
A numerical study on slip flow in packed hyper-porous media inside micro-channels using differential transformation method
}

\author{
Puyan Saffari $^{1}$, Esmaeel Fatahian ${ }^{2 *}$, Peyman Saffari ${ }^{2}$, Hossein Fatahian ${ }^{2}$ \\ ${ }^{1}$ PhD student, Department of Mechanical Engineering, Lahijan Branch, Islamic Azad University, Guilan, Iran \\ ${ }^{2}$ PhD student, Department of Mechanical Engineering, Nour Branch, Islamic Azad University, Mazandaran, Iran \\ *Corresponding author E-mail: esmaeelfatahian@gmail.com
}

\begin{abstract}
Velocity slip boundary condition in parallel-plate micro-channels filled with hyper-porous media is studied using differential transformation method (DTM) in order to find an analytical approximate solution. The results focus on slip flow regime (i.e., for Knudsen numbers in the range $10^{-3}<\mathrm{Kn}<10^{-1}$ ). The Darcy-Brinkman-Forchheimer model is applied to study the effect of nonlinear drag term further boundary-friction effects on hydrodynamic of gas flow in micro-channels. The results show that DTM results are in good agreement with numerical ones. Also, it is observed that decreasing the value of Darcy number flattens the velocity profile while this trend is opposite for decreasing the Forchheimer number. Also, increasing the value of $\alpha$ causes to increase the velocity slip at the wall.
\end{abstract}

Keywords: Slip Flow; Hyper-Porous Media; Differential Transformation Method.

\section{Introduction}

For the first time Nield and Kuznetsov in 2006 brought to the notice the problem of forced convection with slip-flow in a channel or duct occupied by a hyper-porous medium saturated by a rarefied gas. [1] They use the linear Darcy-Brinkman model and found exact analytical solution for velocity and temperature profiles as well as Nusselt number using velocity slip and temperature jump boundary conditions. However, the problem becomes more complex when it comes to consider the effect of non-linear drag form term. In the other word when one applies BrinkmanForchheimer model it is impossible to find an exact analytical solution. Hence, researchers have to solve the equation using numerical methods. For example, in a recent year Haddad et.al. in 2006 and 2007 used the finite difference technique to study laminar forced convection of gaseous slip flow in a parallel-plate and circular micro-channel filled with Darcy-Brinkman-Forchheimer porous media. [2], [3] Therefore, inducement of presenting an analytical solution for the problem of forced convection in a porous-saturated channel with slip flow and temperature jump could be its importance in easier analysis of micro and nano channel flow systems with heat transfer applications, because of having a smooth solution which can be integrated easily for finding temperature distribution and Nusselt number. Differential transformation method is one of the interesting semi-analytical methods which yield to an accurate approximate solution in compare with the exact solution and was first proposed by Zhou in 1986. [4]
Until recently many researchers including Kundu and Barman in 2010, Yaghoobi and Torabi in 2011, Rashidi in 2009, Rashidi and Keimanesh in 2010, Rashidi and Sadri in 2010 used this method to find approximate analytical solutions for nonlinear differential equations in many fields of their study. [5-9] Chamkha in 1996 and 1998 has detailed the influence of a magnetic field on an electrically conducting fluid in the neighborhood of a cone, a wedge or near a stagnation point. [10], [11] More recently, Desseaux in 1999 analyzed the influence of a magnetic field over a laminar viscous flow in a semi-porous channel. All these problems and phenomena are modeled by ordinary or partial differential equations. [12]

The present work focuses on presenting an analytical approximate solution based on differential transformation method for the problem of slip flow in a micro-channel packed with Darcy-BrinkmanForchheimer hyper-porous media, in order to consider the effect of inertia effects on hydrodynamic aspects. The merit of obtaining an analytical approximate is that we can easily integrate or derivate the solution easily which is different from numerical one.

\section{Mathematical formulation}

Figure 1 shows steady laminar gas flow between parallel-plate micro-channel filled with a porous medium subject to the velocity slip boundary conditions. 


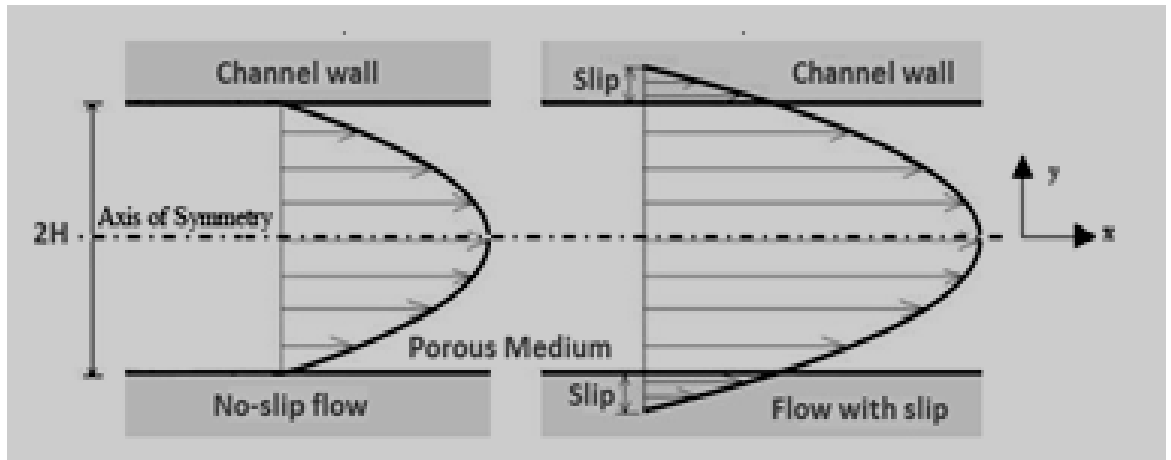

Fig. 1: Definition Sketch.

The momentum equations are:

$\not d^{2} u^{*}{ }_{d y}^{* 2}-\frac{\mu u^{*}}{K}-\frac{c_{F} \rho}{\sqrt{K}} u^{* 2}+G=0$,

The non-dimensional form of momentum equation is as follows:

$M \frac{d^{2} u}{d y^{2}}-\frac{u}{D a}-\Gamma u^{2}+1=0$.

By defining the following dimensionless variables:

$y=\frac{y^{*}}{H}$,

$u=\frac{\not a^{*}}{G H^{2}}, D a=\frac{K}{H^{2}}, M=\frac{\not c_{0}}{\mu}, \Gamma=\frac{{ }^{c_{F}} \rho G H^{4}}{\sqrt{K} \mu^{2}}$.

Where $y^{*}$ is transverse coordinate, $\mathrm{H}$ is the half channel distance, $\not t o$ is the effective viscosity, $u^{*}$ is the velocity, $\mathrm{G}$ is the negative pressure gradient, $\rho$ is the fluid density, $c_{F}$ is the inertial coefficient and $K$ is the permeability of porous medium. $\mathrm{D}_{\mathrm{a}}$ is the Darcy number; $\mathrm{M}$ is the viscosity ratio and $\Gamma$ is the Forchheimer number. The associated boundary conditions for solving Equation (1) are as follows:

$u=-\alpha \frac{d u}{d y}$ at $y=1$, And $\frac{d u}{d y}=0$ at $y=0$.

$\alpha=\frac{2-\sigma_{v}}{\sigma_{v}} K n$

Where $\mathrm{Kn}$ is the Knudsen number which is the ratio of the meanfree-path to a characteristic macroscopic length scale, and is in the range $10^{-3}<\mathrm{Kn}<10^{-1}$ for slip flow regime. $\sigma_{v}$ is a quantity that is called the momentum accommodation coefficient.

\section{Analytical solution based on DTM}

Now we apply the differential transformation method into Equation (4). Taking the differential transform of Equation (4) with respect to $Y$ gives: (5)

$$
\begin{aligned}
& (k+2)(k+1) U(k+2)-c_{1} U(k) \\
& -c_{2}\left(\sum_{l=0}^{k} U(l) U(k-l)\right)+c_{3}=0
\end{aligned}
$$

Where

$c_{1}=\frac{1}{M D a}, c_{2}=\frac{\Gamma}{M}, c_{2}=\frac{1}{M}$.

From boundary condition in Equation (4) that we have it in point $y=0$ and exerting transformation:

$U(1)=0$

The other boundary conditions are considered as follow:

$U(0)=a$

Where $a$ is constant and we will calculate it with considering another boundary condition in Equation (2) in point $Y=1$.

Accordingly, from a process of inverse differential transformation, in this problem we calculated $U(k+2)$ from Equation (5) as following:

$U(2)=\frac{1}{2} c_{1} a+\frac{1}{2} c_{2} a^{2}-\frac{1}{2} c_{3}$

$U(3)=0$

$U(4)=\frac{1}{24} c_{1}^{2} a+\frac{1}{8} c_{1} c_{2} a^{2}-\frac{1}{24} c_{1} c_{3}+\frac{1}{12} c_{2}^{2} a^{3}-\frac{1}{12} c_{2} c_{3} a$

The above process may be continued further. Substituting Eq. (8) into the main equation based on DTM, the closed form of the solutions is obtained as:

$u(y)=U(1) y+U(2) y^{2}+U(3) y^{3}+U(4) y^{4}+$

We substitute the boundary condition from Equation (4) into Equation (9) and use Newton-Raphson iterative technique for determination of unknown value of $a$. Substituting for $a$ into Eq. (9) so we determine $u(y)$.

The calculations reported in this paper use $n=10$ which was found to be sufficient to give an accurate solution. 


\section{Results and discussion}

First of all, the analytical results obtained by DTM are validated with numerical results of the software MAPLE 12, and then the parametric study on velocity profile demonstrates how changing a key dimensionless parameter affect the velocity profile. The re-

sults are presented at $\mathrm{M}=1$ which is more common and $\sigma_{v}=0.7$.

As can be seen in Figure 2 there is an excellent agreement between the DTM and those of numerical (Finite difference method) results at different values of key parameters which confirms the robustness of DTM solution.

Figure 3 indicates the effect of Darcy number on the dimensionless velocity profile. As expected decreasing the value of $\mathrm{Da}$ makes a more uniform velocity distribution. This is due to the fact that lower Da associates lower permeability of the porous medium and lower fluid penetration.

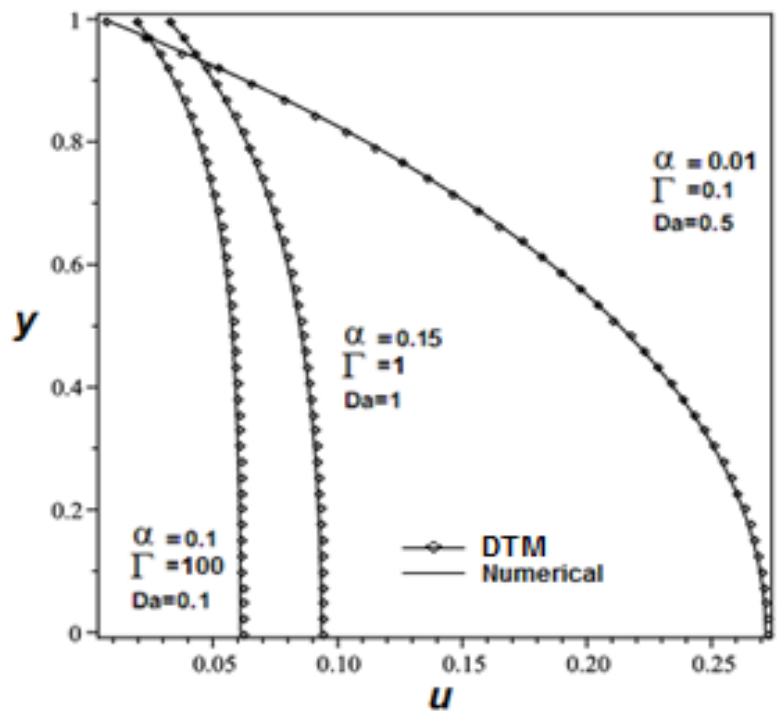

Fig. 2: Validation of DTM Solution with Numerical Results.

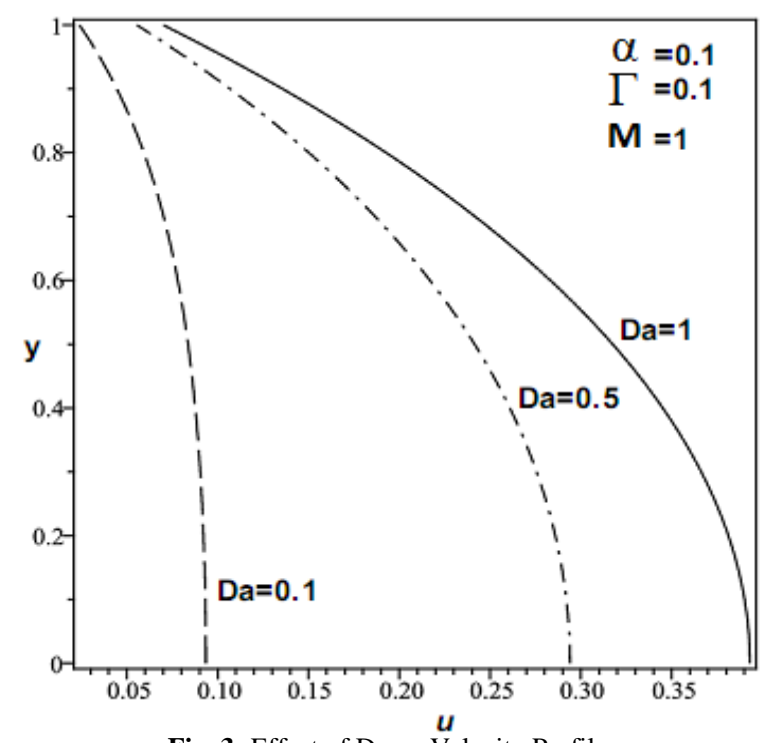

Fig. 3: Effect of Da on Velocity Profile.

Figure 4 indicate the effect of $\alpha$ which is a proportion of Knudson number on velocity profile at $\mathrm{Da}=0.1$ and $\Gamma=0.1$. It is clear from the figure that, increasing the value of $\alpha$ leads to increasing the velocity slip at the wall. The reason is that, increasing in $\mathrm{Kn}$ can be due to an increase in the mean free path of the molecules which yields larger flow rates through the channel due to decrease of the retarding effect at the wall. Effect of forchheimer number on velocity is shown in figure 5 .
According to the figure with increasing the value of $\Gamma$, fluid velocity decreases and its profile becomes flatter. This is due to the fact that higher forchheimer number means increasing the inertia effects and leads to increasing the resistance to the flow and tends the flow pattern to a more slug-like flow.

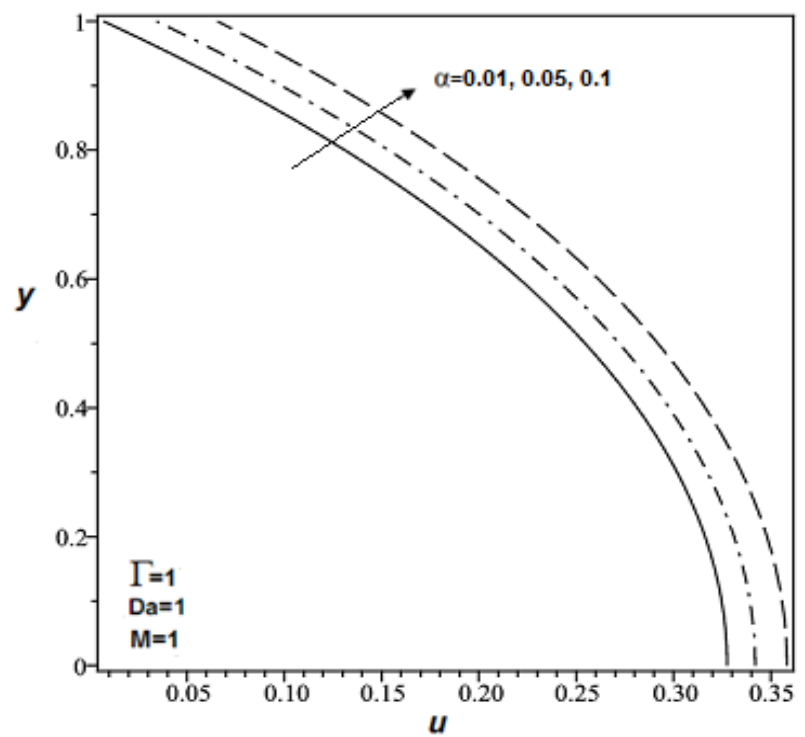

Fig. 4: Effect of Slip Flow Parameter on Velocity.

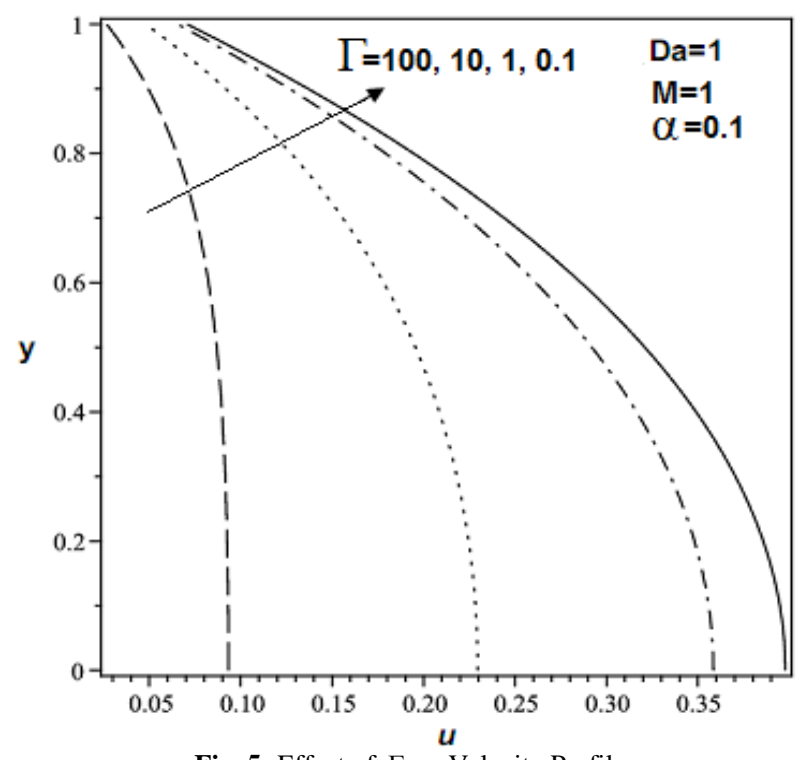

Fig. 5: Effect of $\Gamma$ on Velocity Profile.

\section{Conclusion}

Slip flow in parallel plates micro-channels filled with a hyper porous media is studied analytically by use of differential transformation method for solving modified extended Darcy-BrinkmanForchheimer model. Analytical results were compared with numerical solution and effects of different dimensionless parameters were studied on velocity profile. The results show that decreasing the value of Da makes a more uniform velocity distribution.

The reason is the lower Da associates lower permeability of the porous medium. With increasing the value of $\Gamma$, velocity of fluid decreases and its profile becomes flatter. This is due to the fact that higher forchheimer number leads to increase the inertia effects and increase the resistance to the flow. Also, increasing the value of $\alpha$ leads to increasing the velocity slip at the wall. The reason is that, increasing in $\mathrm{Kn}$ can be due to an increase in the mean free path of the molecules which causes larger flow rates through the channel. 


\section{References}

[1] Nield, D. A., and A. V. Kuznetsov. "Forced convection with slipflow in a channel or duct occupied by a hyper-porous medium saturated by a rarefied gas." Transport in porous media 64.2 (2006): 161-170. http://dx.doi.org/10.1007/s11242-005-2341-x.

[2] Haddad, O. M., and Yazan Taamneh. "Hydrodynamic and thermal behavior of gas flow in micro-channels filled with porous media." Journal of Porous Media 9.5 (2006).

[3] Haddad, O. M., M. A. Al-Nimr, and M. S. Sari. "Forced convection gaseous slip flow in circular porous micro-channels." Transport in $\begin{array}{llll}\text { Porous } & \text { Media } & 70.2 & \text { (2007): }\end{array}$ http://dx.doi.org/10.1007/s11242-006-9093-0.

[4] Zhou, J. K. "Differential transformation and its applications for electrical circuits." (1986): 1279-1289.

[5] Kundu, B., and D. Barman. "Analytical study on design analysis of annular fins under dehumidifying conditions with a polynomial relationship between humidity ratio and saturation temperature." International Journal of Heat and Fluid Flow 31.4 (2010): 722-733. http://dx.doi.org/10.1016/j.ijheatfluidflow.2010.01.010.

[6] Yaghoobi, Hessameddin, and Mohsen Torabi. "The application of differential transformation method to nonlinear equations arising in heat transfer." International Communications in Heat and Mass $\begin{array}{llll}\text { Transfer } & 38.6 & \text { (2011): } & \text { 815-820. }\end{array}$ http://dx.doi.org/10.1016/j.icheatmasstransfer.2011.03.025.

[7] Rashidi, M. M. "The modified differential transform method for solving MHD boundary-layer equations." Computer Physics Com$\begin{array}{lll}\text { munications } & 180.11 & \text { (2009): }\end{array}$ http://dx.doi.org/10.1016/j.cpc.2009.06.029.

[8] Rashidi, Mohammad Mehdi, and Mohammad Keimanesh. "Using differential transform method and padé approximant for solving mhd flow in a laminar liquid film from a horizontal stretching surface." Mathematical Problems in Engineering 2010 (2010). http://dx.doi.org/10.1155/2010/491319.

[9] Rashidi, M. M., and S. M. Sadri. "Solution of the laminar viscous flow in a semi-porous channel in the presence of a uniform magnetic field by using the differential transform method." Int. J. Contemp. Math. Sciences 5.15 (2010): 711-720.

[10] Chamkha, Ali J. "Non-Darcy hydromagnetic free convection from a cone and a wedge in porous media." International communications in heat and mass transfer 23.6 (1996): 875-887. http://dx.doi.org/10.1016/0735-1933(96)00070-X

[11] Chamkha, Ali J. "Hydromagnetic plane and axisymmetric flow near a stagnation point with heat generation." International Communications in Heat and Mass Transfer 25.2 (1998): 269-278. http://dx.doi.org/10.1016/S0735-1933(98)00014-1.

[12] Desseaux, André. "Influence of a magnetic field over a laminar viscous flow in a semi-porous channel." International journal of engineering science $37.14 \quad$ (1999): $1781-1794$. http://dx.doi.org/10.1016/S0020-7225(99)00003-8 